\title{
The Role of Cytokines in Nephrotic Syndrome
}

\author{
Elham Ahmadian $\mathbb{D}^{1},{ }^{1}$ Yalda Rahbar Saadat $\mathbb{D}^{1},{ }^{1}$ Elaheh Dalir Abdolahinia, ${ }^{2}$ Milad Bastami, ${ }^{3}$ \\ Mohammadali M. Shoja, ${ }^{4}$ Sepideh Zununi Vahed $\mathbb{D}^{1},{ }^{1}$ and Mohammadreza Ardalan $\mathbb{D}^{1}$ \\ ${ }^{1}$ Kidney Research Center, Tabriz University of Medical Sciences, Tabriz, Iran \\ ${ }^{2}$ Research Center for Pharmaceutical Nanotechnology, Biomedicine Institute, Tabriz University of Medical Sciences, Tabriz, Iran \\ ${ }^{3}$ Noncommunicable Diseases Research Center, Fasa University of Medical Sciences, Fasa, Iran \\ ${ }^{4}$ Clinical Academy of Teaching and Learning, Ross University School of Medicine, Miramar, FL, USA
}

Correspondence should be addressed to Sepideh Zununi Vahed; sepide.zununi@gmail.com and Mohammadreza Ardalan; ardalan34@yahoo.com

Received 26 May 2021; Revised 16 January 2022; Accepted 27 January 2022; Published 9 February 2022

Academic Editor: Matilde Otero-Losada

Copyright (c) 2022 Elham Ahmadian et al. This is an open access article distributed under the Creative Commons Attribution License, which permits unrestricted use, distribution, and reproduction in any medium, provided the original work is properly cited.

\begin{abstract}
Idiopathic nephrotic syndrome (INS) is an important primary glomerular disease characterized by severe proteinuria. Evidence supports a role for T cell dysfunction in the pathogenesis of INS. Glucocorticoids are the primary therapy for INS; however, steroid-resistant NS (SRNS) patients are at a higher risk of drug-induced side effects and harbor poor prognosis. Although the exact mechanism of the resistance is unknown, the imbalances of T helper subtype 1 (Th1), Th2, and regulatory T cells (Tregs) and their cytokines may be involved in the pathogenesis of glucocorticoid responsiveness. Up to now, no confirmed biomarkers have been able to predict SRNS; however, a panel of cytokines may predict responsiveness and identify SRNS patients. Thus, the introduction of distinctive cytokines as novel biomarkers of SRNS enables both preventions of drug-related toxicity and earlier switch to more effective therapies. This review highlights the impacts of $\mathrm{T}$ cell population imbalances and their downstream cytokines on response to glucocorticoid responsiveness state in INS.
\end{abstract}

\section{Introduction}

Idiopathic nephrotic syndrome (INS) is a clinical definition, described by extreme proteinuria due to podocyte injury and foot process effacement. Focal segmental glomerulosclerosis (FSGS) and minimal change disease (MCD) are the two most important light microscopic pictures of this glomerular disease and the most common causes of INS in both adults and pediatrics. Despite the current lack of knowledge in a comprehensive understanding of the disease mechanism, the response to glucocorticoids and/or other immunosuppressant agents indicates the primary involvement of the immune system. The current observations are in favor of the association of $\mathrm{T}$ regulatory (Treg), $\mathrm{T}$ helper subtype 1 (Th1), and T helper subtype 2 (Th2) imbalances and their related cytokines in the pathogenesis of INS $[1,2]$.

The activation of the inflammation cascades is heterogeneous and diverse in FSGS or MCD. A sequential produc- tion of proinflammatory cytokines leads to a systemic inflammatory response initiated with the synthesis of IL-1 and TNF- $\alpha$ (TNF), which, in turn, escalates the generation of IL- 6 . The production of cytokines stimulates the formation of acute-phase proteins such as haptoglobin, haemopexin, or C-reactive protein (CRP), suPAR (soluble urokinase-type plasminogen activator receptor), $\alpha-1$ antitrypsin, and fibrinogen in the liver. Alpha-1 antitrypsin and fibrinogen are more sensitive to IL- 6 stimulation while others are generally synthesized in response to IL-1 [3]. Different studies have reported the plausible connection between cytokine production and proteinuria in INS $[4,5]$. However, conflicting results have been obtained when the serum levels of the major cytokines and acute-phase proteins are measured in patients with NS [6-8].

Glucocorticoids are the standard initial pharmacological regimen in INS, which block the production of cytokines in both immune and nonimmune cells effectively and result in 
remissions in approximately $85-90 \%$ of pediatric cases. However, individuals exhibit different degrees of glucocorticoid responsiveness and variable patterns of relapses [9]. Glucocorticoids represent a key index of outcomes, and drug-resistant patients pose a challenge to clinicians. Furthermore, glucocorticoid dependency is observed in about $40-50 \%$ of the responders who are at high risk of therapyassociated unwanted effects [10]. Indeed, no clinical test is available to predict steroid resistance and/or dependence.

The response to glucocorticoids has been considered as the key variable in long-term outcomes of FSGS and MCD patients [11]. The potential effects of glucocorticoids highlight the possible role of cytokines in determining the drug response. SRNS patients without podocyte genetic defects may also respond to other immunosuppressive agents, such as cyclosporine, tacrolimus, and mycophenolate.

\section{Factors Involved in SRNS}

The impacts of epigenetic, pharmacogenetic, and genetic factors on the pathogenesis of SRNS have been comprehensively reviewed previously [12-15]. In the presence of podocyte cytoskeletal-related mutations, glucocorticoids are ineffective at restoring normal podocyte function. About $30 \%$ of SRNS patients have mutations in one of the podocyte-expressed genes. Circulating factors, such as serum urokinase-type plasminogen activator receptor or cardiotrophin-like cytokine 1 , are another proposed pathogenic mechanism [16]. Cytokines are reported to modulate the glucocorticoid responses in NS [17-19]. In the following sections, we provide reported articles linking imbalanced $\mathrm{T}$ cell populations and their dysregulated cytokines to SRNS.

\section{Cytokines Affect the Responses to Glucocorticoid Therapy}

Because of the controversial reports regarding the cytokine patterns of Th1/Th2, subtypes, and glucocorticoid response, studies are aimed at introducing these possible biomarkers $[20,21]$. In the following sections, we focus on the impacts of $\mathrm{T}$ cell population imbalance and its downstream cytokines on SRNS.

3.1. T Cell Population Imbalance in SRNS. Despite conflicting evidence, the imbalance between Th1, Th2, and Treg cells has been associated with the incidence of SRNS. If glucocorticoids mediate alterations in T cells' population and their cytokine profile, then steroid-sensitive NS (SSNS) and SRNS patients should have differences in their T cell populations. It has been demonstrated that Th1/Treg and Th2/Treg ratios are higher in SRNS compared to SSNS patients and healthy individuals, while Th1/Th2 ratios are similar among the groups. A higher ratio of Treg in comparison with Th1 and Th2 is connected with glucocorticoid sensitivity, while the reverse ratio is associated with SRNS [22]. Guimarães et al. made a study on a group of children with INS (steroid-sensitive (16 boys/9 girls) and steroid-resistant 8/6) and 10 healthy controls. They observed downregulated levels of adhesion molecules (integrin, CD18) and higher levels
$(48 \%)$ of Treg (TCD $4^{+}$CTLA $-4^{+}$FoxP $^{+}$) in the steroidsensitive group [23]. NS patients who are more prone to relapse or do not respond to glucocorticoids show an immunological switching from Th2 to Th1 [24]. In line with these findings, serum cytokines shift toward the Th1 pattern in FSGS patients [24]. Additionally, in a study on a group of INS children (29 SSNS and 14 SRNS children, aged between 2 and 19 years), higher levels of Th1 cytokines (e.g., IL-2) have been found in their serum and urine samples, whereas elevated Th2-related cytokine (i.e., IL-4) generation was associated with long-term remission [5]. However, both glucocorticoid sensitive and resistant patients show similar levels of Th1- and Th2-associated cytokines; these differences might be due to different lymphocyte stimuli [4]. Stachowski and coworkers also reported similar results and concluded that the $\mathrm{CD}^{+} \mathrm{T}$ cell-related cytokine pattern and the distribution of particular $\mathrm{T}$ cell subsets, including suppressor-effector $\left(\mathrm{CD} 45 \mathrm{RA}^{+} \mathrm{CD}^{+}\right)$, suppressor-inducer $\left(\mathrm{CD} 45 \mathrm{RA}^{+} \mathrm{CD} 4^{+}\right)$, and memory cells $\left(\mathrm{CD} 45 \mathrm{RO}^{+} \mathrm{CD} 4^{+}\right)$, might predict the patients' sensitivity to glucocorticoids at the onset of NS [25]. The importance of the Th1/Th2 balance has been confirmed by increased levels of Th1 cytokines (including IL-2, soluble IL-2 receptor (sIL-2R), and IFN- $\gamma$ ) in SSNS patients during relapse [26]. Hence, assessing the balance of Th1/Th2 could be valuable in predicting glucocorticoid responsiveness.

Effective glucocorticoid therapy has been shown to restore the functional balance of the Th-17/Treg population in MCD patients [27]. Moreover, primary glucocorticoid therapy has reduced $\mathrm{CD} 8^{+} \mathrm{T}$, Th2, and $\mathrm{CD} 4^{+} \mathrm{Th} 1$ cells in NS patients. Accordingly, glucocorticoid therapy effectively diminishes $\mathrm{CD}^{+} \mathrm{T}$, Th2, and $\mathrm{CD}^{+}$Th1 cells in new-onset pediatric NS cases [28].

Response to glucocorticoid therapy in children with NS is influenced by the levels of IL-13 and TNF- $\beta$ (lymphotoxin-alpha). Elevated levels of TNF- $\beta$ are observed in SRNS patients after treatment while SSNS cases developed higher levels of IL-13. Increased levels of IL-13 may be in connection with TNF- $\beta$ downregulation in SSNS patients since the latter is suppressed via Th2 cytokines [29]. Interaction between TNF receptor and soluble lymphotoxin-alpha promotes inflammatory responses. $\mathrm{T}$ cell deviation towards the Th2 population in NS patients might also be linked to the overproduction of IL-13. These findings propose that Th1-dominant patients might develop glucocorticoid-resistance, while increased IL-13 and Th2 phenotypes are in favor of a satisfactory outcome, and glucocorticoid responsiveness. Therefore, alteration in Th1 and Th2 populations and subsequent changes in IL-13/TNF- $\beta$ cytokines balance substantially affect NS pathophysiology in children [29].

3.2. T Cell Resistance to Glucocorticoids. Particular mediators influence $\mathrm{T}$ cell resistance to glucocorticoids. For example, IL-2 and IL-4 promote lymphocyte glucocorticoid resistance during an in vitro study [30]. In addition, nuclear factor- $\kappa \mathrm{B}$ $(\mathrm{NF}-\kappa \mathrm{B})$ and transcription factor activator protein-1 (AP-1) are pivotal mediators of proinflammatory cytokine generation and have been found to interfere with glucocorticoid functions on T cells [31]. In this context, the glucocorticoid 
receptor $\alpha(\mathrm{GR} \alpha)$ suppresses AP-1 activity via direct proteinprotein interaction with a c-Jun subunit of the AP-1 family [32]. Interestingly, it is documented that AP-1 modulates the structure of basal chromatin and increases the accessibility of GR and its binding to proinflammatory genes [33]. Hence, it appears that the interactions between AP-1 and glucocorticoids are far more complicated.

3.2.1. NF- $\kappa B$ Signaling. NF- $\kappa \mathrm{B}$ is a transcription factor that regulates the transcription of genes participating in inflammation. Sun and colleagues reported that the overexpression of $\mathrm{NF}-\kappa \mathrm{B}$ in the juvenile Sprague-Dawley rat model of nephrotic syndrome induces the expression of inflammatory cytokines (IL-1 and IL-6), increases blood urea nitrogen and creatinine levels, and exacerbates renal injury [34]. NF- $\kappa \mathrm{B}$ as a member of the Rel family contains two subunits ( $\mathrm{p} 50$ and p65) [35]. The binding of NF- $\kappa \mathrm{B}$ to the endogenous $\mathrm{I} \kappa \mathrm{B}$ family proteins makes it inactive. The release of NF- $\kappa \mathrm{B}$ from $\mathrm{I} \kappa \mathrm{B}$ occurs upon antigenic stimulation and subsequent phosphorylation of $\mathrm{I} \kappa \mathrm{B}$ via $\mathrm{I} \kappa \mathrm{B}$ kinases $\alpha$ and $\beta$. SRNS patients have a lower level of NF- $\kappa$ B p 65 subunit in the whole-cell lysates, prepared from the peripheral mononuclear blood cells (PMBC) compared to glucocorticoid-sensitive cases [36]. Both lower levels of NF- $\kappa \mathrm{B}$ p 65 and GR $\alpha$ are connected with poor glucocorticoid responses in some patients with INS. This difference is more prominent in those experiencing relapses [36]. However, both SSNS and SRNS patients express similar levels of the p50 subunit. The translocation of the NF- $\kappa \mathrm{B}$ p50 subunit into the nucleus is essential for the interaction of $\mathrm{NF}-\kappa \mathrm{B}$ with glucocorticoids, and the absence of such translocation impairs the ability of GRs to inhibit immune functions and NF- $\kappa \mathrm{B}$ transcriptional activity, inducing glucocorticoid resistance $[31,32]$.

The expression of IL-2 is also increased during the relapse of both SSNS and SRNS patients in comparison with controls. These results indicated alterations in the $\mathrm{T}$ cell populations between untreated SRNS and SSNS patients. The upregulation of IL-2 and down-regulation of NF- $\kappa \mathrm{B}$ p65 subunits are possible mechanisms of glucocorticoid resistance in NS [37]. It has been reported that three mechanisms are involved in this process. First, the absence of required protein-protein interactions, especially among $\mathrm{GR} \alpha$ and p65 subunits. Second, disturbances in nuclear export of NF- $\kappa \mathrm{B}$ dimers, and third plunged affinity of NF$\kappa \mathrm{B}$ for the glucocorticoid-stimulated leucine zipper that acts as an inhibitor of NF- $\kappa$ B nuclear translocation [37]. In SRNS patients, steroid-based treatment might fail by enhancing $\mathrm{NF}-\kappa \mathrm{B}$ function, which would worsen disease by elevating transcription of inflammatory cytokines [38].

\section{Cytokines in SRNS}

The prevalence of relapses in NS has been associated with the serum levels of particular cytokines (Table 1). Some researchers have attempted to identify urinary, plasma, and salivary cytokine-based biomarkers for SRNS in children [39-41]. Both SSNS and SRNS patients have shown suppressed levels of IL-5, IL-7, IL-13, IFN- $\gamma$, and TNF after glucocorticoid administration. Furthermore, SRNS patients have been shown to have higher levels of MIP-1 $\beta$, IL-17A, IL-5, and INF- $\gamma$ in comparison with SSNS cases in preand posttreatment specimens. Agrawal et al. studied the plasma profile of cytokines in children [SSNS $(n=26)$ and SRNS $(n=14)$ ] aged between 18 months and 18 years before and after (7 weeks) treatment with glucocorticoids. Using a bead-based fluorescence assay, the profiling of 27 cytokines was evaluated on a Luminex Technology platform (Waltham, MA). Different levels of 13 plasma cytokines were observed between SSNS versus SRNS before therapy. Three cytokines (IL-7, IL-9, and MCP-1) exhibited ROC (receiver operating characteristic) values of $0.846,0.64$ sensitivity, and 0.84 specificity and could differentiate children with SRNS from those with SSNS at the disease onset. Furthermore, their results detected significant reductions in cytokine levels (e.g., IFN- $\gamma$, TNF, IL-5, IL-7, and IL-13) in response to glucocorticoid treatment in SSNS compared to SRNS patients. The authors proposed that glucocorticoid therapy decreases cytokine production by $\mathrm{CD} 4^{+} \mathrm{Th} 1$ cells, Th2 cells, and $\mathrm{CD}^{+}$cells in children with new-onset NS [28] (Figure 1).

Increased IL-8 concentration has been associated with relapses in NS [42] and antibodies against IL-8 could neutralize the ability of mononuclear cells to trigger albuminuria in the Wistar rat model [43]. Moreover, surged amount of IL- $1 \beta$, IL- 6 , and IL- 8 has been observed in INS relapses compared to healthy controls or remission in children [44]. In addition, IL-4, IL-6, and TNF polymorphisms have been in connection with glucocorticoid responsiveness in INS children [45]. The activation of TGF- $\beta 1$ has been reported in SRNS cases, which further develop chronic kidney disease (CKD). FSGS patients have shown higher levels of urinary TGF- $\beta 1$ compared to MCD patients. However, urinary TGF- $\beta 1$ has not been validated as a glucocorticoid responsiveness biomarker [19]. Elevated serum levels of IL-6, haptoglobin, and haemopexin are also independent markers of glucocorticoid resistance in FSGS and MCD patients [3].

$\mathrm{T}$ cell expressing inflammatory cytokines, plasma macrophage migration inhibitory factor (MIF), and urinary MCP-1 are increased during persistent proteinuria in pediatric SRNS [41]. The role of glomerular macrophages and the underlying mechanism of macrophage-related glucocorticoid resistance have not been clarified. The substantial connection between urinary MCP-1 and IL-6 or interferoninducible protein-10 (IP-10) suggests that the MCP-1stimulated macrophages can generate IL-6 or IP-10 after recruitment to the glomeruli, which might then lead to tissue damage and enrollment of other immune cells [46, 47].

4.1. MIF. MIF has been considered as a suitable marker for glucocorticoid responsiveness among 48 evaluated cytokines. According to cytokine analysis, the increased plasma concentrations of MIF (cutoff concentration of MIF > 501 $\mathrm{pg} / \mathrm{ml}$ ) at diagnosis could identify NS children at high risk of glucocorticoid resistance. Low levels (MIF mean concentration $124.5 \mathrm{pg} / \mathrm{ml}$ in healthy controls vs. $466.1 \mathrm{pg} / \mathrm{ml}$ in INS patients) of this cytokine could also successfully discriminate INS patients from controls [48]. MIF displays proinflammatory activities as a result of interactions with $\mathrm{T}$ cells 


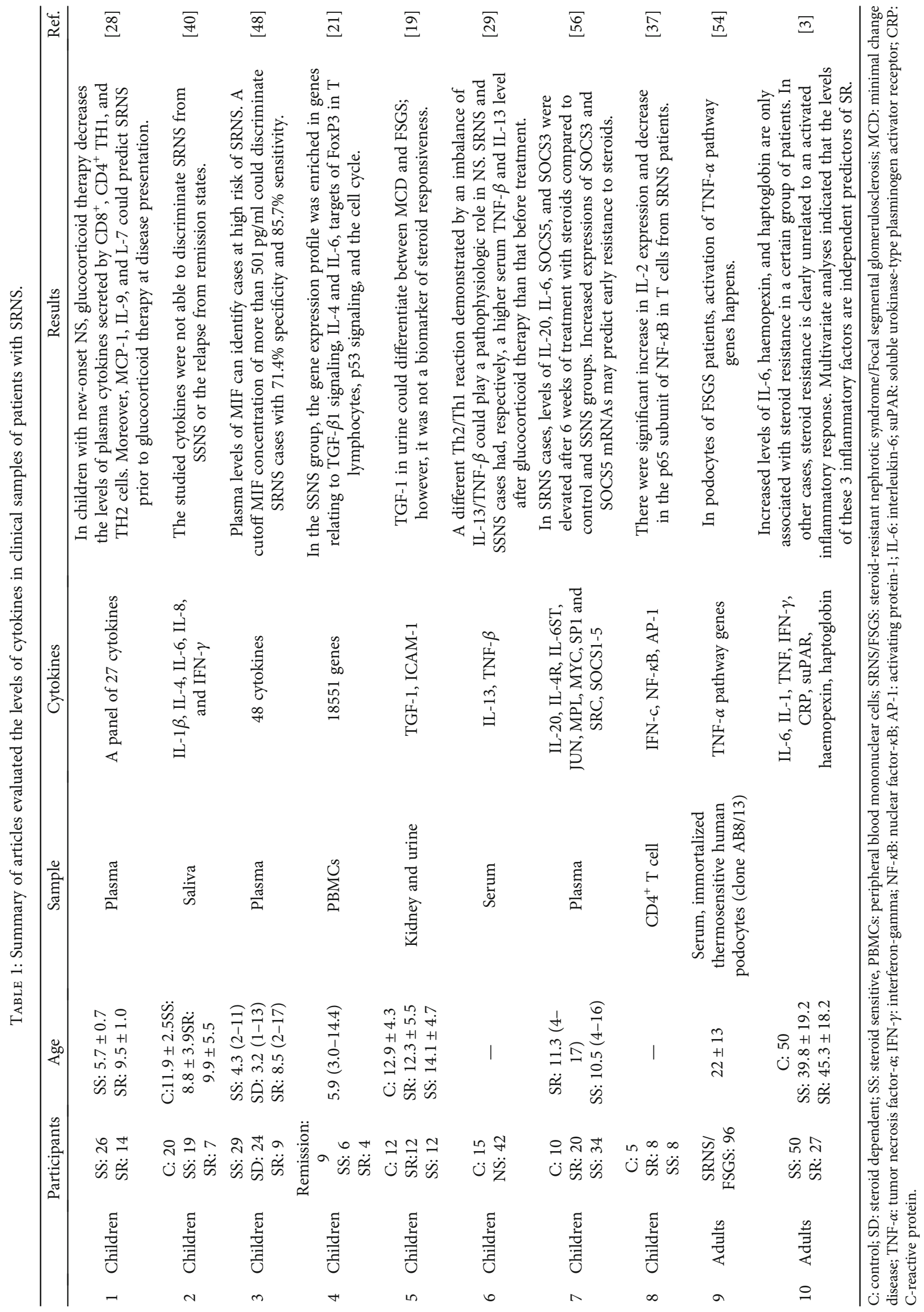



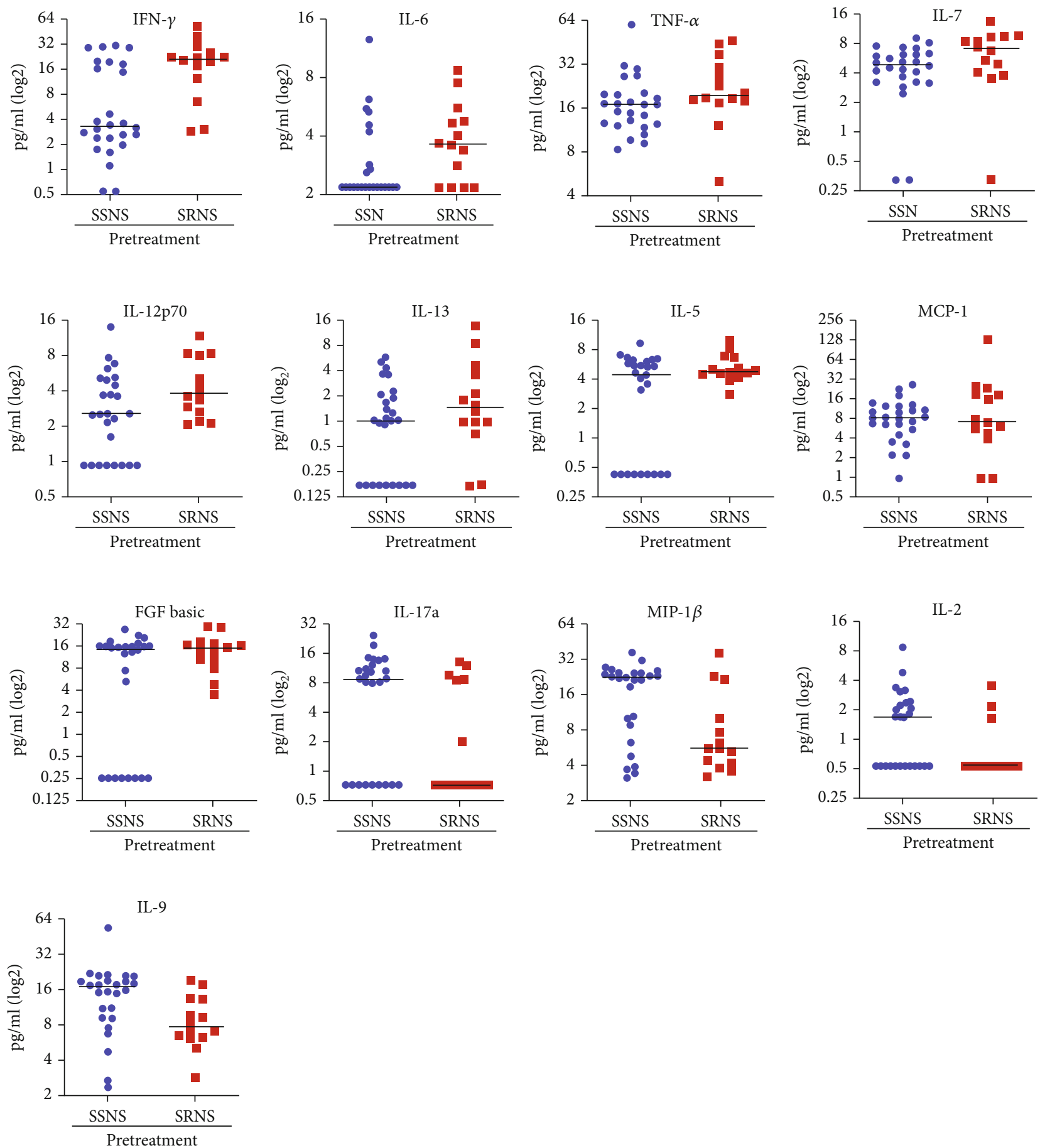

FIGURE 1: Cytokines can identify the SRNS cases before therapy. Different levels of 13 plasma cytokines were observed between SSNS versus SRNS before therapy, three of which (MCP-1, IL-9, and L-7) had values to discriminate SRNS from SSNS prior to glucocorticoid therapy with ROC value $=0.84,0.64$ sensitivity, and 0.84 specificity. FGF: fibroblast growth factor; MCP-1: monocyte chemoattractant protein-1; MIP-1 $\beta$ : macrophage inflammatory protein- $1 \beta$; SSNS: steroid-sensitive nephrotic syndrome; SRNS: steroid-resistant nephrotic syndrome; TNF: tumor necrosis factor; ROC: receiver operating characteristic. Adapted from Ref. [28] with permission. The reference [28] article is available under the Creative Commons CC-BY-NC-ND license.

and macrophages. Glucocorticoids decrease the formation of inflammatory mediators; however, they accelerate MIF release from T cells and macrophages [49]. Then, MIF counterregulates the suppressor effects of glucocorticoids on proinflammatory cytokines [50]. Although the underlying mechanisms are not completely known, it has been postulated that MIF interferes with the function of glucocorticoid under an inflammatory condition mediated by NF- $\kappa \mathrm{B}$ dependent manner. Glucocorticoids prevent the NF- $\kappa$ B activation through the induction of $\mathrm{I} \kappa \mathrm{B} \alpha$ synthesis, whereas MIF 
enhances the translocation of NF- $\kappa \mathrm{B}$ to the nucleus [51]. Furthermore, MIF potently induces the extracellular signal-regulated kinase- (ERK-) 1 and ERK-2 pathways, which in turn, activate the intracellular isoform of phospholipase A2 (PLA2) and lead to the liberation of arachidonic acid [52]. Glucocorticoids are recognized blockers of PLA2 stimulation, and this impact is countered by MIF. Additionally, to suppress the transcription of proinflammatory genes, glucocorticoids can raise the degradation of these mRNAs; moreover, this has been revealed to be linked to the inhibitory effect of MIF on glucocorticoids. Although there are inadequate data to elucidate the proinflammatory functions of MIF entirely, the mentioned mechanisms could describe its impact on glucocorticoidrelated immunosuppression $[48,53]$.

4.2. TNF. In kidney glomeruli of patients with FSGS/SRNS, activation of the TNF pathway was observed [54]. TNF is an inflammatory cytokine produced by infiltrating/circulating macrophages and monocytes. The proposed TNF mechanisms of action includes (1) leukocyte recruitment to the glomerular damage site, (2) stimulation of growth factors and cytokines, and (3) generation of oxygen radicals. Consequently, glomerular endothelial damage, apoptosis, and albumin permeability could be the result of those TNFmediated adverse effects [55]. The intrinsic activation of the TNF signaling pathway leads to podocyte damage that can be reversed by the TNF blockader [54].

4.3. Suppressors of Cytokine Signaling. Suppressors of Cytokine Signaling (SOCS) prohibit Signal Transducer and Activator of Transcription (STAT) phosphorylation via blocking Janus Kinases (JaKs), and the effects of glucocorticoids on the JaK/STAT signaling cascade in children with SRNS and SSNS have been investigated. Accordingly, IL-6, IL-20, SOCS3, and SOCS5 were significantly higher in plasma samples of SRNS patients in comparison with SSNS cases. Moreover, the authors suggested the potential role of SOCS3 and SOCS5 mRNA levels as predicting factors of glucocorticoid resistance in patients with NS [56]. Furthermore, substantial lower methylation of one region of the SOCS3 promoter was observed in SRNS participants versus SSNS and normal controls $[56,57]$.

4.4. Other Cytokines. The activation of $\mathrm{T}$ lymphocytes and release of IFN- $\gamma$, IL-4, and IL-2 have been seen in SSNS children with relapse [7]. The plasma level of IL-8 has significantly been in connection with IL-4 and IL-13 in all stages of SSNS in children. Likewise, during the active phase, increased levels of IL-13, IL-4, TNF, and IgE were significantly seen in pediatric SSNS compared to patients in remission and controls [58]. It is deemed that a type-2 cytokine production succeeds in children with active SSNS, and this kind of immune response is closely correlated with the expression of IL-18 [6]. Moreover, serum levels of IL-18 are associated with both IL-4 and IL-13 in pediatric SSNS patients [59]. However, it is also reported that increased levels of IL-18 after therapy can be involved in the SRNS development [60].

\section{Treatment}

The goal of SRNS therapy is inducing complete remission; however, even partial remission may have clinical benefits. For cases with nongenetic-based SRNS, treatment with calcineurin inhibitors (tacrolimus and ciclosporin) is the standard of care therapy and $70 \%$ of them attain a partial or complete remission. The renin-angiotensin inhibitors as antihypertensive and antiproteinuric are quintessential for decreasing proteinuria [61]. Proinflammatory cytokines derived from immune cells promote the formation of angiotensin II (Ang II) both systemically and locally. Production of angiotensinogen by inflammatory cytokines is suggested as a key mechanism for the development of Ang IIdependent high blood pressure [62]. Nonresponding patients to calcineurin inhibitors or immunosuppressives are at risk for ESRD [61].

Epigenetic modification by targeting histone deacetylases (HDACs) are a promising therapeutic approach in NS. Histone deacetylase inhibitors (HDACi) play an important role in treating CKD due to their anti-fibrotic, antiinflammatory, and immunosuppressive activities. HDACi inhibits HDACs, remodels the structure of proteins in transcription factor complexes, and causes modifications in gene transcription by removing the acetyl groups from the lysine amino acid on histone. Thus, HDACi enhances chromatin condensation and exerts a repressor effect on transcription. It is a promising intervention for targeting glomerular sclerosis and fibrosis as important pathologic features of fibrosis and CKD progression both in FSGS and INS patients. Moreover, evidence from various research has demonstrated an irregular expression of HDACs involved in renal fibrosis and glomerulosclerosis which are common pathological features of NS [63]. A combination of HDACi, vorinostat with an ACE inhibitor benazepril in an animal model of nephropathy could significantly reduce proteinuria and kidney injury via modulating different signaling cascades such as NF- $\kappa \mathrm{B}$, IL-1, TGF- $\beta$, MAPK, and apoptosis machinery [66].

\section{Conclusion}

Alterations in cytokine patterns in INS may contribute to proteinuria and glomerular injury and influence therapeutic interventions. Thus, the identification of distinct cytokines as novel biomarkers of SRNS at the early diagnosis can benefit patients by both enabling the prevention of glucocorticoid toxicity and directing to earlier switch to more effective therapeutic options. Understanding the molecular mechanisms involved in SRNS and the development of molecular-based diagnosis and predictive biomarkers would have a significant value in the management of SRNS patients in years to come.

\section{Data Availability}

No original data were used in this study. 


\section{Conflicts of Interest}

The authors declare that they have no conflicts of interest.

\section{Authors' Contributions}

SZV and MA developed the idea. EA and YRS reviewed the literature and prepared the first draft. MA and SZV revised the manuscript. All authors have read and approved the final manuscript.

\section{Acknowledgments}

This study was supported by the Kidney Research Center, Tabriz University of Medical Sciences, Tabriz, Iran (Grant No. \#69028).

\section{References}

[1] J. J. Candelier and H. K. Lorenzo, "Idiopathic nephrotic syndrome and serum permeability factors: a molecular jigsaw puzzle," Cell and Tissue Research, vol. 379, no. 2, pp. 231243, 2020.

[2] E. T. McCarthy, M. Sharma, and V. J. Savin, "Circulating permeability factors in idiopathic nephrotic syndrome and focal segmental glomerulosclerosis," Clinical Journal of the American Society of Nephrology, vol. 5, no. 11, pp. 2115-2121, 2010.

[3] N. Roca, C. Martinez, E. Jatem, A. Madrid, M. Lopez, and A. Segarra, "Activation of the acute inflammatory phase response in idiopathic nephrotic syndrome: association with clinicopathological phenotypes and with response to corticosteroids," Clinical Kidney Journal, vol. 14, no. 4, pp. 12071215, 2021.

[4] A. P. Carlotti, P. B. Franco, L. L. Elias et al., "Glucocorticoid receptors, in vitro steroid sensitivity, and cytokine secretion in idiopathic nephrotic syndrome," Kidney International, vol. 65, no. 2, pp. 403-408, 2004.

[5] V. Daniel, Y. Trautmann, M. Konrad, A. Nayir, and K. Schärer, "T-Lymphocyte populations, cytokines and other growth factors in serum and urine of children with idiopathic nephrotic syndrome," Clinical Nephrology, vol. 47, no. 5, pp. 289-297, 1997.

[6] N. Printza, F. Papachristou, V. Tzimouli, A. Taparkou, and F. Kanakoudi-Tsakalidou, "IL-18 is correlated with type-2 immune response in children with steroid sensitive nephrotic syndrome," Cytokine, vol. 44, no. 2, pp. 262-268, 2008.

[7] T. J. Neuhaus, M. Wadhwa, R. Callard, and T. M. Barratt, "Increased IL-2, IL-4 and interferon-gamma (IFN-gamma) in steroid-sensitive nephrotic syndrome," Clinical and Experimental Immunology, vol. 100, no. 3, pp. 475-479, 1995.

[8] T. Kanai, H. Shiraishi, T. Yamagata et al., "Th2 cells predominate in idiopathic steroid-sensitive nephrotic syndrome," Clinical and Experimental Nephrology, vol. 14, no. 6, pp. 578-583, 2010.

[9] R. J. Hogg, R. J. Portman, D. Milliner, K. V. Lemley, A. Eddy, and J. Ingelfinger, "Evaluation and management of proteinuria and nephrotic syndrome in children: recommendations from a pediatric nephrology panel established at the National Kidney Foundation conference on proteinuria, albuminuria, risk, assessment, detection, and elimination (PARADE)," Pediatrics, vol. 105, no. 6, pp. 1242-1249, 2000.
[10] D. Hahn, E. M. Hodson, N. S. Willis, and J. C. Craig, "Corticosteroid therapy for nephrotic syndrome in children," The Cochrane Database of Systematic Reviews, vol. 18, no. 3, 2015.

[11] M. Nakayama, R. Katafuchi, T. Yanase, K. Ikeda, H. Tanaka, and S. Fujimi, "Steroid responsiveness and frequency of relapse in adult-onset minimal change nephrotic syndrome," American Journal of Kidney Diseases, vol. 39, no. 3, pp. 503512, 2002.

[12] S. M. Hejazian, S. Zununi Vahed, H. Moghaddas Sani et al., "Steroid-resistant nephrotic syndrome: pharmacogenetics and epigenetic points and views," Expert Review of Clinical Pharmacology, vol. 13, no. 2, pp. 147-156, 2020.

[13] S. M. Hosseiniyan Khatibi, M. Ardalan, S. Abediazar, and S. Zununi Vahed, "The impact of steroids on the injured podocytes in nephrotic syndrome," The Journal of Steroid Biochemistry and Molecular Biology, vol. 196, article 105490, 2020.

[14] Y. Rahbar Saadat, S. M. Hejazian, Z. Nariman-Saleh-Fam et al., "Glucocorticoid receptors and their upstream epigenetic regulators in adults with steroid-resistant nephrotic syndrome," BioFactors, vol. 46, no. 6, pp. 995-1005, 2020.

[15] S. Z. Vahed, H. M. Sani, S. Rajabzadeh et al., "The importance of genetic study in steroid-resistant nephrotic syndrome," Journal of Renal Injury Prevention, vol. 8, no. 4, pp. 271-282, 2019.

[16] A. Widiasta, K. Wahyudi, H. Nugrahapraja, Y. Sribudiani, and D. Rachmadi, "The unique difference between serum level of soluble urokinase plasminogen activator receptor (suPAR) in steroid-resistant nephrotic syndrome children treated with an alkylating agent and calcineurin inhibitors," Journal of Comprehensive Pediatrics, vol. 12, no. 2, article e109912, 2021.

[17] Y. S. Youn, H. H. Lim, and J. H. Lee, "The clinical characteristics of steroid responsive nephrotic syndrome of children according to the serum immunoglobulin E levels and cytokines," Yonsei Medical Journal, vol. 53, no. 4, pp. 715-722, 2012.

[18] H.-K. Yap, W. Cheung, B. Murugasu, S.-K. Sim, C.-C. Seah, and S. C. Jordan, "Th1 and Th2 cytokine mRNA profiles in childhood nephrotic syndrome: evidence for increased IL-13 mRNA expression in relapse," Journal of the American Society of Nephrology, vol. 10, no. 3, pp. 529-537, 1999.

[19] R. P. Woroniecki, I. F. Shatat, K. Supe, Z. Du, and F. J. Kaskel, "Urinary cytokines and steroid responsiveness in idiopathic nephrotic syndrome of childhood," American Journal of Nephrology, vol. 28, no. 1, pp. 83-90, 2007.

[20] H. Stone, B. Magella, and M. R. Bennett, "The search for biomarkers to aid in diagnosis, differentiation, and prognosis of childhood idiopathic nephrotic syndrome," Frontiers in Pediatrics, vol. 7, p. 404, 2019.

[21] H. G. Kang, H. Seo, J. H. Lim et al., "Markers of disease and steroid responsiveness in paediatric idiopathic nephrotic syndrome: whole-transcriptome sequencing of peripheral blood mononuclear cells," The Journal of International Medical Research, vol. 45, no. 3, pp. 948-963, 2017.

[22] A. Jaiswal, N. Prasad, V. Agarwal et al., "Regulatory and effector $\mathrm{T}$ cells changes in remission and resistant state of childhood nephrotic syndrome," Indian Journal of Nephrology, vol. 24, no. 6, pp. 349-355, 2014.

[23] F. T. L. Guimarães, R. N. Ferreira, G. E. A. Brito-Melo et al., "Pediatric patients with steroid-sensitive nephrotic syndrome have higher expression of T regulatory lymphocytes in comparison to steroid-resistant disease," Frontiers in Pediatrics, vol. 7, p. 114, 2019. 
[24] N. Futrakul, P. Butthep, S. Patumraj, and P. Futrakul, "Glomerular endothelial dysfunction and altered cytokines in severe nephrosis," Nephron, vol. 86, no. 2, p. 199, 2000.

[25] J. Stachowski, C. Barth, J. Michałkiewicz et al., "Th1/Th2 balance and CD45-positive T cell subsets in primary nephrotic syndrome," Pediatric Nephrology, vol. 14, no. 8, pp. 779-785, 2000.

[26] G. Lama, I. Luongo, G. Tirino, A. Borriello, C. Carangio, and M. E. Salsano, "T-lymphocyte populations and cytokines in childhood nephrotic syndrome," American Journal of Kidney Diseases, vol. 39, no. 5, pp. 958-965, 2002.

[27] L. L. Liu, Y. Qin, J. F. Cai et al., "Th17/Treg imbalance in adult patients with minimal change nephrotic syndrome," Clinical Immunology, vol. 139, no. 3, pp. 314-320, 2011.

[28] S. Agrawal, M. E. Brier, B. A. Kerlin et al., "Plasma cytokine profiling to predict steroid resistance in pediatric nephrotic syndrome," Kidney International Reports, vol. 6, no. 3, pp. 785-795, 2021.

[29] Y. L. Tain, T. Y. Chen, and K. D. Yang, "Implications of serum TNF-beta and IL-13 in the treatment response of childhood nephrotic syndrome," Cytokine, vol. 21, no. 3, pp. 155-159, 2003.

[30] J. C. Kam, S. J. Szefler, W. Surs, E. R. Sher, and D. Y. Leung, "Combination IL-2 and IL-4 reduces glucocorticoid receptorbinding affinity and T cell response to glucocorticoids," Journal of Immunology, vol. 151, no. 7, pp. 3460-3466, 1993.

[31] L. Panda, A. Gheware, R. Rehman et al., "Linoleic acid metabolite leads to steroid resistant asthma features partially through NF- $\kappa$ B," Scientific Reports, vol. 7, no. 1, p. 9565, 2017.

[32] K. A. Smoak and J. A. Cidlowski, "Mechanisms of glucocorticoid receptor signaling during inflammation," Mechanisms of Ageing and Development, vol. 125, no. 10-11, pp. 697-706, 2004.

[33] S. C. Biddie, S. John, P. J. Sabo et al., “Transcription factor AP1 potentiates chromatin accessibility and glucocorticoid receptor binding," Molecular Cell, vol. 43, no. 1, pp. 145-155, 2011.

[34] X. M. Sun, X. H. Sun, Z. Y. Li, C. J. Yuan, and J. Wu, "Expression of NF- $\kappa$ B in juvenile rats with nephrotic syndrome and its effects on inflammatory changes and renal injury," European Review for Medical and Pharmacological Sciences, vol. 23, no. 9, pp. 4010-4016, 2019.

[35] A. Oeckinghaus and S. Ghosh, "The NF-kappaB family of transcription factors and its regulation," Cold Spring Harbor Perspectives in Biology, vol. 1, no. 4, article a000034, 2009.

[36] K. Szilagyi, L. Podracka, N. E. Franke, J. Mojzis, and L. Mirossay, "A new link between steroid resistance, glucocorticoid receptor and nuclear factor kappa B p65 in idiopathic nephrotic syndrome," Neuro Endocrinology Letters, vol. 30, no. 5, pp. 629-636, 2009.

[37] D. H. Aviles, V. Matti Vehaskari, J. Manning, A. C. Ochoa, and A. H. Zea, "Decreased expression of T-cell NF-kappaB p65 subunit in steroid-resistant nephrotic syndrome," Kidney International, vol. 66, no. 1, pp. 60-67, 2004.

[38] C. Dawson, A. Dhanda, B. Conway-Campbell, A. Dimambro, $\mathrm{S}$. Lightman, and C. Dayan, "NF $\mathrm{B}$ and glucocorticoid receptor activity in steroid resistance," Journal of Receptor and Signal Transduction Research, vol. 32, no. 1, pp. 29-35, 2012.

[39] F. T. L. Guimarães, G. Melo, T. M. Cordeiro et al., "T-Lymphocyte-expressing inflammatory cytokines underlie persistence of proteinuria in children with idiopathic nephrotic syndrome," Jornal de Pediatria, vol. 94, no. 5, pp. 546-553, 2018.
[40] D. Polak, Y. Borovitz, D. Clyman-Levy et al., "Salivary cytokines in children with nephrotic syndrome versus healthy children: a comparative study," Journal of Clinical Medicine, vol. 9, no. 9, p. 2691, 2020.

[41] Y. Matsumoto, Y. Ikezumi, T. Kondo et al., "Urinary monocyte chemotactic protein 1 as a predictive marker of steroid responsiveness in children with idiopathic nephrotic syndrome," Fujita Medical Journal, vol. 4, no. 1, pp. 17-22, 2018.

[42] E. H. Garin, D. K. Blanchard, K. Matsushima, and J. Y. Djeu, "IL-8 production by peripheral blood mononuclear cells in nephrotic patients," Kidney International, vol. 45, no. 5, pp. 1311-1317, 1994.

[43] E. H. Garin, P. Laflam, and L. Chandler, "Anti-interleukin 8 antibody abolishes effects of lipoid nephrosis cytokine," Pediatric Nephrology, vol. 12, no. 5, pp. 381-385, 1998.

[44] A. A. Al-Eisa, M. Al Rushood, and R. J. Al-Attiyah, "Urinary excretion of IL- $1 \beta$, IL- 6 and IL- 8 cytokines during relapse and remission of idiopathic nephrotic syndrome," Journal of Inflammation Research, vol. 10, pp. 1-5, 2017.

[45] T. Jafar, S. Agrawal, A. A. Mahdi, R. K. Sharma, S. Awasthi, and G. G. Agarwal, "Cytokine gene polymorphism in idiopathic nephrotic syndrome children," Indian Journal of Clinical Biochemistry, vol. 26, no. 3, pp. 296-302, 2011.

[46] G. D. Han, K. Suzuki, H. Koike et al., "IFN-inducible protein10 plays a pivotal role in maintaining slit-diaphragm function by regulating podocyte cell-cycle balance," Journal of the American Society of Nephrology, vol. 17, no. 2, pp. 442-453, 2006.

[47] R. O'Leary, H. Penrose, K. Miyata, and R. Satou, "Macrophagederived IL-6 contributes to ANG II-mediated angiotensinogen stimulation in renal proximal tubular cells," American Journal of Physiology, vol. 310, no. 10, pp. F1000-F1007, 2016.

[48] E. Cuzzoni, R. Franca, S. De Iudicibus et al., "MIF plasma level as a possible tool to predict steroid responsiveness in children with idiopathic nephrotic syndrome," European Journal of Clinical Pharmacology, vol. 75, no. 12, pp. 1675-1683, 2019.

[49] T. Calandra and T. Roger, "Macrophage migration inhibitory factor: a regulator of innate immunity," Nature Reviews. Immunology, vol. 3, no. 10, pp. 791-800, 2003.

[50] T. Calandra, J. Bernhagen, C. N. Metz et al., "MIF as a glucocorticoid-induced modulator of cytokine production," Nature, vol. 377, no. 6544, pp. 68-71, 1995.

[51] J. M. Daun and J. G. Cannon, "Macrophage migration inhibitory factor antagonizes hydrocortisone-induced increases in cytosolic IkappaBalpha," American Journal of Physiology, vol. 279, no. 3, pp. R1043-R1049, 2000.

[52] R. A. Mitchell, C. N. Metz, T. Peng, and R. Bucala, "Sustained mitogen-activated protein kinase (MAPK) and cytoplasmic phospholipase A2 activation by macrophage migration inhibitory factor (MIF). Regulatory role in cell proliferation and glucocorticoid action," The Journal of Biological Chemistry, vol. 274, no. 25, pp. 18100-18106, 1999.

[53] T. Roger, A. L. Chanson, M. Knaup-Reymond, and T. Calandra, "Macrophage migration inhibitory factor promotes innate immune responses by suppressing glucocorticoid-induced expression of mitogen-activated protein kinase phosphatase-1," European Journal of Immunology, vol. 35, no. 12, pp. 3405-3413, 2005.

[54] C. F. Chung, T. Kitzler, N. Kachurina et al., "Intrinsic tumor necrosis factor- $\alpha$ pathway is activated in a subset of patients with focal segmental glomerulosclerosis," PLoS One, vol. 14, no. 5, article e0216426, 2019. 
[55] A. Ortiz, C. Bustos, J. Alonso et al., "Involvement of tumor necrosis factor-alpha in the pathogenesis of experimental and human glomerulonephritis," Advances in Nephrology from the Necker Hospital, vol. 24, pp. 53-77, 1995.

[56] D. Ostalska-Nowicka, M. Smiech, M. Jaroniec et al., "SOCS3 and SOCS5 mRNA expressions may predict initial steroid response in nephrotic syndrome children," Folia Histochemica et Cytobiologica, vol. 49, no. 4, pp. 719-728, 2011.

[57] K. Zaorska, P. Zawierucha, D. Ostalska-Nowicka, and M. Nowicki, "SOCS3 is epigenetically up-regulated in steroid resistant nephrotic children," Acta Biochimica Polonica, vol. 63, no. 1, pp. 131-138, 2016.

[58] D. M. Youssef, R. M. Elbehidy, A. S. El-Shal, and L. M. Sherief, "T helper 1 and T helper 2 cytokines in atopic children with steroid-sensitive nephrotic syndrome," Iranian Journal of Kidney Diseases, vol. 9, no. 4, pp. 298-305, 2015.

[59] S. A. Shalaby, H. M. Al-Edressi, S. A. El-Tarhouny, M. Fath ElBab, and M. A. Zolaly, "Type 1/type 2 cytokine serum levels and role of interleukin-18 in children with steroid-sensitive nephrotic syndrome," Arab Journal of Nephrology and Transplantation, vol. 6, no. 2, pp. 83-88, 2013.

[60] H. K. Jiang, G. Luo, and H. Jiang, "Interleukin-18 expression in peripheral blood mononuclear cells in children with steroidresistant nephrotic syndrome," Chinese Journal of Contemporary Pediatrics, vol. 11, no. 5, pp. 337-340, 2009.

[61] K. Tullus, H. Webb, and A. Bagga, "Management of steroidresistant nephrotic syndrome in children and adolescents," The Lancet Child \& Adolescent Health, vol. 2, no. 12, pp. 880-890, 2018.

[62] R. Satou, H. Penrose, and L. G. Navar, "Inflammation as a regulator of the renin-angiotensin system and blood pressure," Current Hypertension Reports, vol. 20, no. 12, p. 100, 2018.

[63] R. Varghese and A. Majumdar, "Current therapies in nephrotic syndrome: HDAC inhibitors, an emerging therapy for kidney diseases," Current Research in Biotechnology, vol. 3, pp. 182-194, 2021. 